\title{
Optimal path analysis for solving nonlinear equations with finite local error
}

\author{
Xiaoxiao Ma*, \\ School of Transportation, Chongqing Vocational College of Transportation, \\ Chongqing, 402247 \\ China \\ Xiaojuan Chen, \\ School of Road, Bridge and Architecture, Chongqing Vocational College of Transportation, \\ Chongqing, 402247 \\ China
}

Received: June 4, 2021. Revised: December 13, 2021. Accepted: January 4, 2022. Published: January 5, 2022.

\begin{abstract}
Because the traditional method of solving nonlinear equations takes a long time, an optimal path analysis method for solving nonlinear equations with limited local error is designed. Firstly, according to the finite condition of local error, the optimization objective function of nonlinear equations is established. Secondly, set the constraints of the objective function, solve the optimal solution of the nonlinear equation under the condition of limited local error, and obtain the optimal path of the nonlinear equation system. Finally, experiments show that the optimal path analysis method for solving nonlinear equations with limited local error takes less time than other methods, and can be effectively applied to practice.
\end{abstract}

Keywords-Constraints, mixed, local error, nonlinear equations, optimization path.

\section{INTRODUCTION}

$\mathrm{T}$ HE solution of nonlinear algebraic equations is a basic and important problem. A large number of practical problems in engineering practice, economics, information security and dynamics are converted into algebraic equations. Therefore, the solution of nonlinear equations becomes an important research content in engineering application and numerical calculation. Nonlinear equations refer to the relationship between dependent variables and independent variables is not linear, this kind of equations are many, such as square relations, logarithmic relations, exponential relations, trigonometric functions, and so on. It is often difficult to obtain accurate solutions for this kind of equations, so approximate solutions are required, and the corresponding approximate solutions are gradually getting more and more attention. Nonlinear optimization is the interdisciplinary operational research and computational mathematics, many practical problems such as national defense, oil exploration, weather forecast, management, finance and other problems can be summarized as nonlinear optimization problems, such as the pattern recognition problems in information science, the portfolio problems in finance, the inversion problems in earth science, etc. These practical problems are usually high dimensional nonlinear problems, so it is of great significance to study efficient methods to solve nonlinear optimization problems. The ill-posed nonlinear observation model is widely used in geodetic measurement and parameter inversion. For example, the Angle observation equation and the edge length observation equation with unknown parameters are both nonlinear functions of the coordinate of the fixed point. There are also a lot of nonlinear least square estimation problems in the field of deformation monitoring and inverse calculation of subgrade and pavement modulus. Because the observation error of modern field measurement is equal to or even less than the linear approximation error. Therefore, the study of the solution theory and method of ill-posed nonlinear observation model has not only theoretical significance, but also important engineering application value.

At present, many research achievements have been made on the solution of nonlinear equations. Since the mathematical models of practical problems are mostly nonlinear equations, and only numerical solutions can be obtained, two kinds of algorithms can be divided into iterative algorithm and intelligent algorithm according to different types of algorithms. Iterative algorithm thought is through the preset initial solution, using some iterative way to get the solution of correction, finally reached the requirements of precision and so on, in which Newton's method and its improved algorithm is the iteration algorithm of a class of nonlinear equations is very important, its main advantage is the convergence speed of 2 order, but the requirements for the initial value is very high, need as much as 
possible close to the exact solution, at the same time each iteration needs computing Jacobi matrix, the relatively large amount of calculation, the solution for a long time, so the intelligent algorithm arises at the historic moment, In this paper, an optimal path method for solving nonlinear equations with local error is designed.

\section{Method And MATERIALS}

Because the traditional method of solving nonlinear equations takes a long time, this paper designs an optimal path analysis method for solving nonlinear equations with limited local error. Through the finite condition of local error, the optimization objective of nonlinear equations is established. According to the optimization objective, the constraint conditions are set, and the optimal path to solve the nonlinear equations under the condition of local error is given.

\section{A. Optimization Objective Function of Nonlinear Equation}

The solution of nonlinear optimization problem is an important part in the study of real life optimization problem. In practical operation, the accuracy of solving one-dimensional subproblems needs to be improved because the one-dimensional accurate line search method can generate a large amount of computation, especially when the initial point is far away from the optimal solution point [1]. If Newton's method and quasi-newton's method are used to solve real problems, their convergence rate does not depend on the precise one-dimensional line search process. Therefore, we only need to ensure that every step of the calculation process of the objective function is optimized.

This paper mainly studies Newton method, quasi-newton method and particle swarm optimization, summarizes the advantages and disadvantages of each algorithm in solving nonlinear equations, and improves the algorithm. For quasi-newton method and particle swarm optimization (PSO) algorithm to solve the problem of the advantages and disadvantages, the hybrid strategy, will be mixed quasi-newton method and particle swarm optimization (PSO), concluded that the new method in solving nonlinear equation, mixed quasi-newton particle swarm algorithm, the method to make up for the disadvantage of the two algorithms to solve the problem alone, the design of the local error under the condition of limited system of nonlinear equations solution optimization path model is shown in Fig. 1:

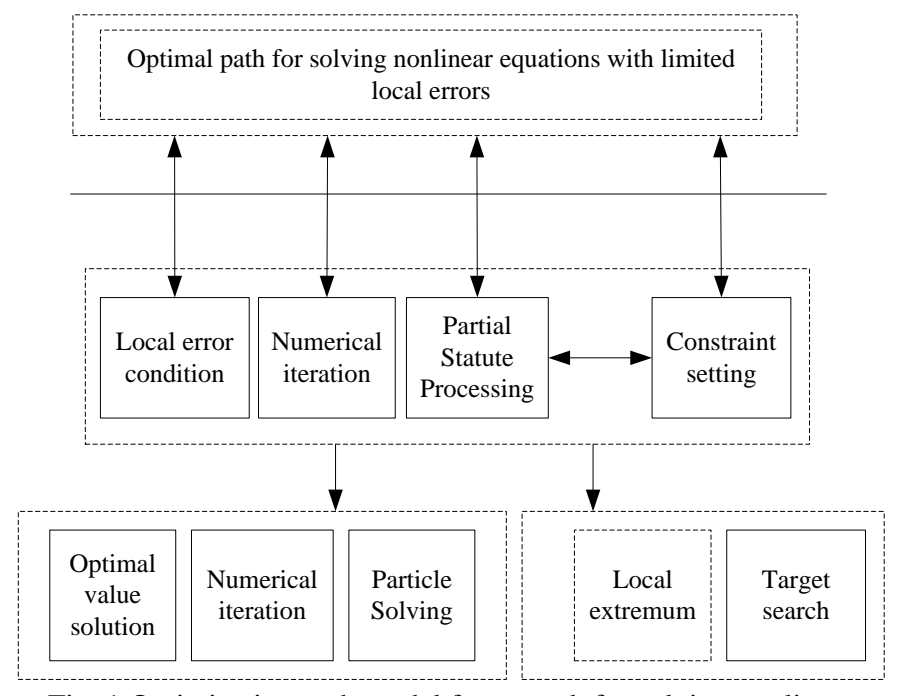

Fig. 1 Optimization path model framework for solving nonlinear equations under local error limit

\section{B. Objective Function Constraint Setting}

Nonlinear least squares problems of numerical iteration algorithm in the iteration process, most of them need to inverse iterative matrix, when the iteration matrix is morbid matrix, as a result of the iterative matrix inversion is not stable, may lead to poor iterative convergence effect, thus the nonlinear least squares problems is not the first qualitative phenomenon. Therefore, combining the characteristics of the nonlinear least square iterative matrix, the ill-posed criterion of the nonlinear least square iterative matrix is established. Based on the conditional number theory, a new method to reduce the conditional number of the iterative matrix is proposed [2]-[5]. Set the optimization goal and set the constraint conditions before optimization, as shown in Table I:

Table I Constraint characteristics table

\begin{tabular}{|c|c|c|}
\hline Index & Content & Method \\
\hline 1 & $\begin{array}{l}\text { Level of abstraction of } \\
\text { information }\end{array}$ & Capturing design intent \\
\hline 2 & $\begin{array}{l}\text { Description of the } \\
\text { constraint model }\end{array}$ & Information expression level \\
\hline 3 & Support sketch design & $\begin{array}{l}\text { Determining the Topological } \\
\text { Structure of Geometric Models } \\
\text { Using Variational Geometry }\end{array}$ \\
\hline 4 & $\begin{array}{l}\text { Support model } \\
\text { modification and } \\
\text { mutation }\end{array}$ & Association semantics \\
\hline 5 & $\begin{array}{l}\text { The modification } \\
\text { process automatically } \\
\text { reflects the designer's } \\
\text { intention }\end{array}$ & $\begin{array}{l}\text { Design environment } \\
\text { automation }\end{array}$ \\
\hline 6 & $\begin{array}{c}\text { Facilitate the conversion } \\
\text { of constraint models to } \\
\text { data models }\end{array}$ & $\begin{array}{l}\text { Modifications and mutations } \\
\text { often drive constraint } \\
\text { relationships through model } \\
\text { size }\end{array}$ \\
\hline
\end{tabular}

The division of constraints is shown in Fig. 2: 


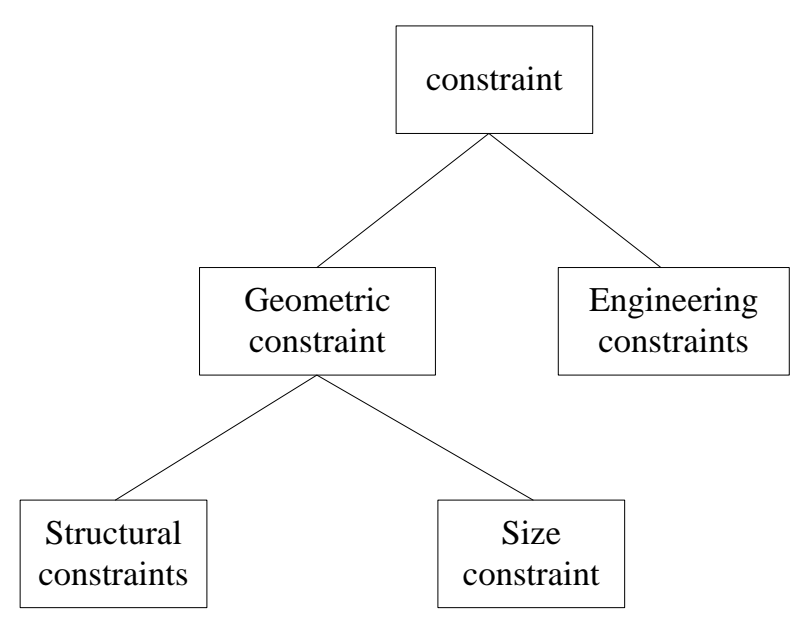

Fig. 2 Constraint partitioning

There are two types of geometric constraints: structural constraints and dimensional constraints. Structural constraint refers to the topological structure relationship between geometric elements, which describes the spatial relative position and connection mode of geometric elements, and its attribute values remain unchanged in the process of parametric design. It is often implicit in two-dimensional engineering drawings, not explicitly given, such as parallel, vertical, tangent and so on. Dimensional constraints are constraints represented by dimensional labels on the graph, such as distance, Angle, etc. Dimensional notation in engineering drawings is a direct and natural descriptor of geometry, thus providing the most appropriate way to modify geometry. The purpose of dimension drive is to modify the graph according to the change of dimension standard value and keep the topological structure relation before and after the graph change unchanged. Contents are shown in Table II:

Table II Optimized content table

\begin{tabular}{|c|c|c|}
\hline Index & Details & Specific method \\
\hline Indicator 1 & $\begin{array}{c}\text { Topological } \\
\text { constraints of general } \\
\text { positional } \\
\text { relationships }\end{array}$ & $\begin{array}{l}\text { Including constraints such as } \\
\text { joint, parallel, and vertical }\end{array}$ \\
\hline Indicator 2 & $\begin{array}{l}\text { Constraints of } \\
\text { various tangent } \\
\text { classes }\end{array}$ & $\begin{array}{l}\text { Constraints such as common } \\
\text { tangent line, common tangent } \\
\text { circle, etc. }\end{array}$ \\
\hline Indicator 3 & $\begin{array}{l}\text { Constraints that } \\
\text { reflect the various } \\
\text { arrangements of } \\
\text { graphics }\end{array}$ & $\begin{array}{l}\text { Such as uniformly distributed } \\
\text { on the circumference, } \\
\text { uniformly distributed on the } \\
\text { rectangle, etc. }\end{array}$ \\
\hline Indicator 4 & $\begin{array}{c}\text { Constraints as } \\
\text { specified in } \\
\text { mechanical drawing } \\
\text { specifications }\end{array}$ & $\begin{array}{l}\text { Such as thread internal } \\
\text { diameter constraint }\end{array}$ \\
\hline Indicator 5 & Symmetry constraint & $\begin{array}{l}\text { Establish the topology } \\
\text { constraints of the graph }\end{array}$ \\
\hline Indicator 6 & $\begin{array}{c}\text { Uniformly } \\
\text { distributed constraint }\end{array}$ & Simplify graphics editing \\
\hline
\end{tabular}

This kind of calculation method also exists in the case of multiple solutions, even under the complete constraint mode, the multiplicity is universal. In the case of multiple solutions, it is necessary to select a set of solutions that conform to the design intention as much as possible from multiple solutions according to certain rules of trade-off. Two basic principles are adopted to deal with the case of multiple solutions in the complete constraint mode:

Principle of invariant topological relations: the relative positions and topological connection relations between geometric elements shall remain unchanged as far as possible, while the principle of minimal shape change: The positions and shape changes of geometric elements shall be minimized as far as possible [6]-[9]. The example where the topological relationship remains unchanged is shown in Fig. 3:

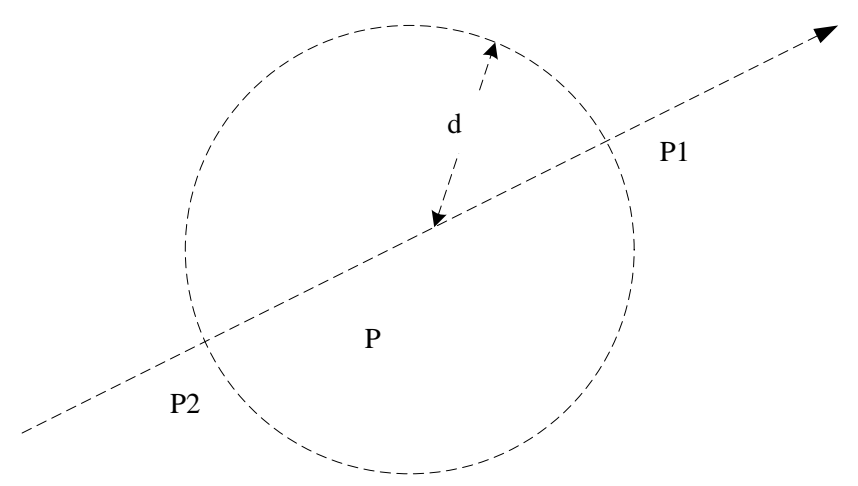

Fig. 3 Example of topology invariant

Based on the above constraints, the rule for finding the optimal solution is generated. Analyze the overall range of local error, replace the weighted coefficient of the solution method of nonlinear equations, and constrain the local error, satisfying the following relation:

$$
G \geq \frac{q}{a r} / \int u
$$

where, $G$ represents the frequent term set of the method of solving nonlinear equations; $\frac{q}{a r} \mathrm{r}$ represents the constraint condition, whose risk is destructive. $\int u$ represents the solution of nonlinear equations to minimize empirical risk.

$\frac{q}{a r}$ as the calculation coefficient of the optimization path of the solution method of nonlinear equations, the local error set is constructed into a sequence of function subsets, so that each local error can be arranged in order from large to small, and the confidence range can be compromised among subsets.

By using this function relation, the convergence characteristics of solutions of nonlinear equations under the condition of local error are calculated:

$$
\phi=\frac{a}{x}[k(x)+q]
$$

where, $\phi$ is the frequent item set under the condition of limited local error [10]-[15]; $\frac{a}{x}$ represents the set matrix; $k(x)$ 
represents the convergence characteristic of the optimal solution of nonlinear equations. $\phi$ as a constraint for the generation of risk identification association rules. If $k(x)$ in the minimum empirical risk in each subset is zero, then the minimum risk permutation is directly selected to determine the constraint conditions. If the number of the subsets in is infinite, a certain empirical risk of the local error condition is taken as the training object for displacement calculation until the optimal solution is obtained.

Through the above process, the optimization goal is set for solving the nonlinear equations with limited local error.

\section{OPTIMIZATION OF SOLUTIONS TO NONLINEAR EQUATIONS UNDER FINITE LOCAL ERROR CONDITIONS}

After the above analysis, the particle swarm optimization algorithm and quasi-newton algorithm are fused to optimize the solution of nonlinear equations under the condition of local error. In particle swarm optimization, the potential solution to each optimization problem is a bird in the search space, called a particle. All particles have a fitness determined by the optimized function, and each particle has a velocity that determines the direction and distance they fly. The particles then follow the current optimal particle through the solution space. The particle swarm optimization algorithm is initialized as a random solution of a bunch of random particles, and then the optimal solution is found through iteration. In each iteration, the particle updates itself by tracking two extremes; The first is the optimal solution found by the particle itself, which is called the individual extremum; The other extreme value is the optimal solution found so far for the whole population. This extreme value is the global extreme value. If you don't use the whole population, but only one part of it as the neighbor of the particle, then the extremum in all the neighbors is the local extremum. Suppose that in a target search space, there is a particle that forms a community, expressed as:

$$
k=\frac{a}{z s} / \sum_{w} a
$$

where, $k$ represents the dimension vector; $\frac{a}{z s}$ represents the optimal location searched so far is called individual extremum; $\sum_{w} a$ represents the global extreme value of the optimal location searched so far for the entire particle swarm.

The whole part is divided into three steps. The first part is the "inertia" or "momentum" part, which reflects the particle's movement "habit" and represents the particle's tendency to maintain its previous speed. The second part is the "cognition" part, which reflects the particle's memory or recall of its own historical experience, and represents the particle's tendency to approach the best position in its own history. The third part is the "society" part, which reflects the group history experience of cooperation and knowledge sharing among particles, and represents the tendency of particles to approach the best historical position of the group or neighborhood. The algorithm flow is as follows:

Step 1: Initialize a group of particles, including random position and velocity;

Step 2: Calculate the fitness value of each particle;

Step 3: For each particle, compare its fitness value with the fitness value of the best historical location. If it is better, take it as the best current location.

Step 4: For each particle, compare its fitness value with the fitness value of the best position globally experienced [16]-[20]. If it is better, take it as the current global best position;

Step 5: If the termination condition is reached and the preset maximum number of iterations is reached, output the current global optimal individual, otherwise let, step 2.

The population covers the whole search space as evenly as possible to improve the global search ability. According to whether the neighborhood of particles is a whole population or not, each particle exchanges information with other particles in the whole population and has a tendency to move to the historical optimal position among all particles. In the initial stage of search, the neighborhood is defined as each particle itself. With the increase of iteration times, the neighborhood scope is gradually extended to the whole population. Performance space refers to the neighborhood divided according to performance indicators such as fitness, objective function value, and as the number of iterations increases, the connection between particles gradually increases, and finally a star topology is formed. The dynamic rank tree is adopted as the neighborhood structure, and the particles with better historical best position are in the upper layer. The velocity of each particle is determined by its own historical best position and the historical best position of the particle at the node above the particle in the rank tree. Whether it is the search of particle swarm in dimension or the cooperative search of multiple particle swarm in different dimensions, the purpose is that each particle can find the learning object that is conducive to fast convergence to the global optimal solution. This learning strategy allows each particle to have more learning objects and to fly over a larger potential space, thus facilitating global search. The speed update formula is:

$$
G=a+\frac{o}{m K_{i}}+\frac{p_{w}}{D}
$$

where, $G$ represents the acceleration factor; $a$ represents uniform random number, $m K_{i}$ represents the learning probability given in advance; $o$ represents the learning object as the best position in its own history; $\frac{p_{w}}{D}$ represents random individuals within a population. Except for the small-scale equation system $\mathrm{W}$ with special form, it is difficult to obtain the exact solution of the nonlinear equation system by the direct solution method. The vast majority of methods for solving nonlinear equations are iterative methods. The iterative method generally linearizes the nonlinear problem and calculates the solution of the linear problem, so as to obtain various forms of iterative sequences. At the same time, in order to ensure that the 
particle can learn from the good object as much as possible and not waste time on the poor object, the above learning object selection process is set to an update interval algebra, during which the learning object remains the same as the last selected learning object. The updated interval algebra Settings are shown in Table III:

Table III Update interval algebra settings

\begin{tabular}{|c|c|c|}
\hline $\begin{array}{l}\text { Particle } \\
\text { expansion }\end{array}$ & $\begin{array}{c}\text { Geometric } \\
\text { constraint } \\
\text { problem } \\
\end{array}$ & Purpose \\
\hline $\begin{array}{c}\text { Assign a } \\
\text { radius to each } \\
\text { particle }\end{array}$ & $\begin{array}{l}\text { Neighborhood } \\
\text { structure }\end{array}$ & $\begin{array}{c}\text { Do not actively change the state } \\
\text { of the neighborhood }\end{array}$ \\
\hline $\begin{array}{l}\text { Assign a } \\
\text { radius to each } \\
\text { particle } \\
\text { Assign a }\end{array}$ & $\begin{array}{l}\text { Macro } \\
\text { neighborhood }\end{array}$ & $\begin{array}{l}\text { Collaborative search in units of } \\
\text { different dimensional } \\
\text { components of space }\end{array}$ \\
\hline $\begin{array}{l}\text { radius to each } \\
\text { particle }\end{array}$ & Search space & Information exchange \\
\hline $\begin{array}{l}\text { Assign a } \\
\text { radius to each } \\
\text { particle }\end{array}$ & Local model & $\begin{array}{l}\text { Learn and communicate within } \\
\text { a given neighborhood }\end{array}$ \\
\hline $\begin{array}{l}\text { Assign a } \\
\text { radius to each } \\
\text { particle }\end{array}$ & $\begin{array}{l}\text { Increase particle } \\
\text { diversity }\end{array}$ & $\begin{array}{l}\text { Each particle introduces a } \\
\text { self-organizing risk index that } \\
\text { is inversely proportional to the } \\
\text { distance of adjacent particles }\end{array}$ \\
\hline $\begin{array}{l}\text { Assign a } \\
\text { radius to each } \\
\text { particle }\end{array}$ & $\begin{array}{l}\text { Distance } \\
\text { judgement }\end{array}$ & $\begin{array}{l}\text { Re-initialize the particle or } \\
\text { push it a certain distance to } \\
\text { reduce the risk }\end{array}$ \\
\hline
\end{tabular}

All the above neighborhood structures, whether micro topology or macro neighborhood, whether it is information exchange in the whole search space or collaborative search based on different dimensional components of the space, do not actively change the neighborhood state. Therefore, there are exceptional constraints, as shown in Fig. 4:

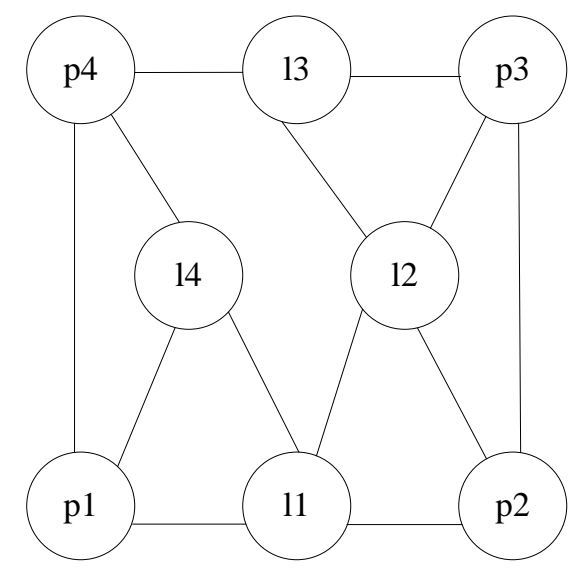

Fig. 4 Geometric constraint diagram

On this basis, particle algorithm and quasi-newtonian algorithm are fused, and the hybrid strategy of selecting these two algorithms can be summarized as shown in Table IV:

Table IVMixed strategies of the two algorithms

\begin{tabular}{|c|c|c|}
\hline Step & Content & Method \\
\hline step 1 & $\begin{array}{c}\text { Fusion of } \\
\text { mechanisms }\end{array}$ & $\begin{array}{c}\text { Track two optimal solutions to update } \\
\text { yourself }\end{array}$ \\
\hline Step 2 & $\begin{array}{l}\text { Organic } \\
\text { integration }\end{array}$ & Strong local search ability \\
\hline Step 3 & $\begin{array}{l}\text { Wide } \\
\text { availability }\end{array}$ & $\begin{array}{c}\text { Removal of previous restrictions when } \\
\text { used alone }\end{array}$ \\
\hline Step 4 & $\begin{array}{c}\text { Features of } \\
\text { parallel } \\
\text { computing }\end{array}$ & $\begin{array}{l}\text { Organic combination of quasi-Newton } \\
\text { method and particle swarm algorithm }\end{array}$ \\
\hline
\end{tabular}

The flow of quasi-newton algorithm is as follows:

Step 1: Initialize a swarm of particles, including random positions and velocities;

Step 2: Calculate the fitness value of each particle;

Step 3: For each particle, compare its fitness value with the fitness value of the best position in history. If it is better, take it as the current best position.

Step 4: For each particle, compare its fitness value with the fitness value of the best position globally experienced. If it is better, take it as the current global best position;

Step 5: If the termination condition is reached and the preset maximum number of iterations is reached, return the current global optimal individual to step 6; Otherwise, go to step 2;

Step 6: If the termination condition is reached (If the preset precision is reached, the end will be reached, and the current result will be output as the optimal solution of the problem; Otherwise continue with quasi-newtonian algorithms.

Finally, the discussion of choosing regular parameters and selecting strategies has been accompanied by the research process of ill-posed problems. From the point of view of computational implementation, the key problem is to determine the correctness to ensure that the solution to the problem is within our tolerance.

In general, the selection methods of regular factors include ridge trace method, generalized cross - verification method, curve method, mean square error minimization method, etc. These methods require the introduction of additional formulas to calculate certain metrics of the iterative process. For example, curve method is used to determine the regular factor. In the measurement adjustment, the method of regular factor selection based on the mean square error criterion has also been studied. Considering the characteristics of the iterative algorithm for nonlinear least square problems and the unsuitability of nonlinear least squares, this chapter proposes two new determination strategies, namely direct search method and interval division method.

The direct search method is adopted, and its contents are shown in Table V:

Table V Search contents by direct search method

\begin{tabular}{ccc}
\hline Content & Classification & Details \\
\hline 1 & Increase the number of & Explore the unknown \\
2 & individuals & space \\
& Spawn new individuals & Maximum accumulated
\end{tabular}


benefits

Bring population

Maintaining group diversity

Since the quantum probability amplitude states that a quantum chromosome carries information about multiple states, the quantum chromosome is in a superposition of multiple determined states before we perform measurements on it. Therefore, a new individual is generated through the probability amplitude of quantum, and its principle is shown in Fig. 5.

According to the above principle, the decision variable is no longer fixed information in a sense, but becomes a kind of information carrying information of different superposition states, so it can bring more abundant population than simply using genetic operation. Second, for some specific problems, it is not convenient to carry out crossover and mutation operations on chromosomes, because the operation will bring a large number of invalid chromosomes. The solutions are either to design special evolutionary operators or to correct invalid chromosomes after evolution, both of which increase the extra cost of the program. In this case, evolutionary manipulation of quantum chromosomes is a good strategy. Therefore, through the above analysis, the conditions for designing and using the genetic quantum algorithm are determined, as shown in Fig. 5:

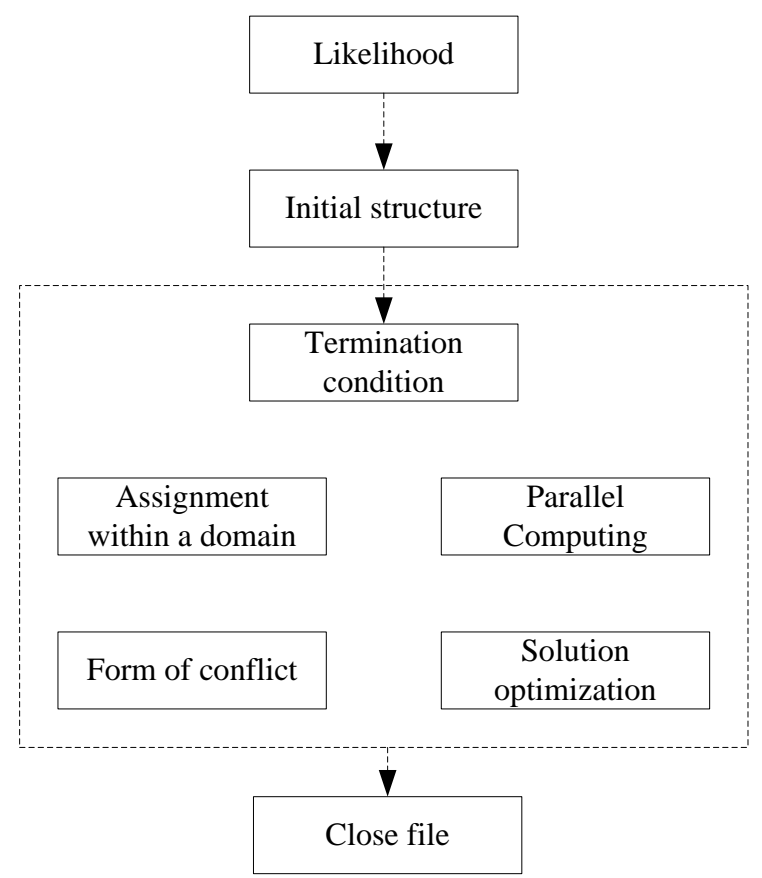

Fig. 5 Conditions for using ga

Combined with the above analysis, the optimization of nonlinear equations under the condition of local error is realized.

\section{EXPERIMENTAL DEMONSTRATION}

Under the effective conditions of the finite nonlinear equations to solve the optimization path analysis method, the partial error process of the design was theoretically verified to verify its practical significance. Through test experiments, the Newton method, the quasi-Newton method and the particle Compared with the group optimization (PSO) method, we compare the Newton algorithm, a kind of H-order convergence algorithm in solving the numerical performance of the root problem of a one-variable function. Secondly, we give the numerical performance of the algorithm in solving general nonlinear equations. Experimental examples are selected from the standard test library, Newton's method W and a class of H-order Newton's algorithm are tested separately, and comparative analysis is done. Numerical experiment code uses the MaticalO. It is written in $\mathrm{O}$ and runs on a personal computer equipped with Intel $3.6 \mathrm{GHz}$ processor, 4GB memory, 64-bit Windows10 operating system. Check the efficiency of solving the four solutions of the nonlinear equation system.

\section{A. Experimental Configuration}

In the Windows 10 operating system, $\mathrm{C}$ language is used for programming, and experiments are conducted on the MATLAB platform. The hardware environment is shown in Table VI:

Table VIExperimental hardware environment settings

Device Environmental parameters
Intel(R) Core(TM) 2 Duo 


\begin{tabular}{ccc}
\hline CPU & $2.94 \mathrm{GHz}$ & Maximum running speed \\
Effective memory & $8 \mathrm{~GB}$ & Maintained during commissioning \\
Initial sampling frequency & $0.8 \mathrm{~Hz}$ & Export experimental data \\
Termination frequency & $0.15 \mathrm{~Hz}$ & Export / print data \\
Bit rate & $0.59 \mathrm{Bps} / \mathrm{s}$ & Guaranteed stable internet connection \\
Microsoft Excel 2003 & & \\
Adobe PDF Reader 7 & $1024 * 768$ or more & \\
Display resolution & $56 \mathrm{kbps}$ & \\
Internet access rate & Seagate Barracuda Series $2 \mathrm{~TB}$ & \\
Mechanical hard drive & Great Wall Dragon 700w & \\
power supply & Kingston A400 Series 240G & \\
SSD & Kingston 3000 frequency 16G $* 2$ & \\
RAM &
\end{tabular}

The experimental software environment is as shown in Table VII

Table VIIExperimental software configuration

\begin{tabular}{ccc}
\hline Serial number & Content & Model \\
\hline 1 & database & mysql \\
2 & Middleware & tomcat \\
\hline
\end{tabular}

\section{B. Experimental Data}

In this experiment, the equations involved in an oil exploration project are selected. The project example is a planar four-bar mechanism. Some schematic diagrams are shown in Fig. 6:

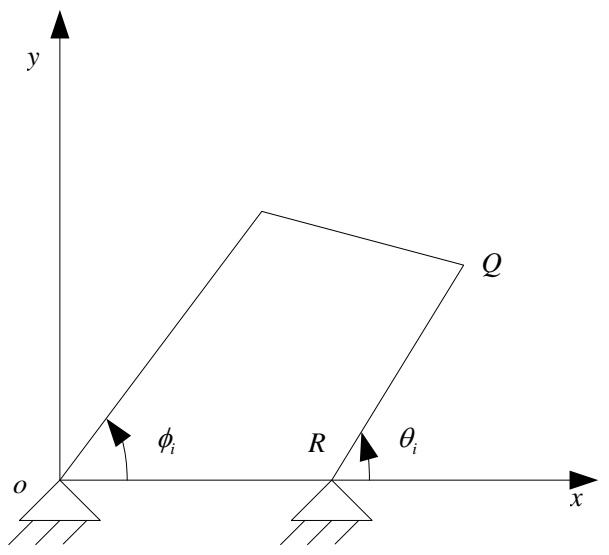

Fig. 6 A schematic diagram of the four-connected mechanism

In engineering practice, the planar four-bar mechanism is widely used, and the kinematic synthesis of the mechanism has multiple solutions. In this project, there are 36 sets of equations during the function synthesis of the planar four-bar mechanism, which are randomly divided into 6 groups and numbered, and one group is randomly selected. The 6 sets of equations involved in this group are shown as follows:

Example 1:

$$
f_{1}(x)=x^{2}-x_{2}+1=0
$$

Example 2:
Example 3:

$$
f_{2}(x)=x_{1}-\cos \left(0.5 \pi x_{2}\right)=0
$$

$$
\left\{\begin{array}{l}
f_{1}(x)=\left(x_{1}-5_{2}\right)^{2}+40 \sin ^{2}\left(10 x_{3}\right)=0 \\
f_{2}(x)=\left(x_{2}-2 x_{3}\right)^{2}+40 \sin ^{2}\left(10 x_{1}\right)=0 \\
f_{3}(x)=\left(3 x_{1}-x_{3}\right)^{2}+40 \sin ^{2}\left(10 x_{2}\right)=0
\end{array}\right.
$$

Example 4:

$$
\left\{\begin{array}{l}
f_{1}(x)=x_{1}^{3}+e^{x 1}+2_{x 3}+x_{3}+1=0 \\
f_{2}(x)=-x_{1}+x_{2}+x_{2}^{3}+2 e^{x 2}-3=0 \\
f_{3}(x)=-2 x_{2}+x_{3}+e^{x 3}+1=0
\end{array}\right.
$$

Example 5:

$$
f_{1}(x)=x_{1}+0.25 x^{2} x_{4}+0.75
$$

Example 6:

$$
f_{1}(x)=x_{6}-x_{1} x_{5}=0
$$

Newton method, quasi-newton method and particle swarm optimization (PSO) were used to solve the above 6 examples respectively, and the solution time of the four methods was compared.

\section{Experimental Scheme}

The field surface runoff monitoring method is used to conduct natural runoff reduction in the selected typical small watershed, fixed point monitoring of rainwater runoff. Simultaneously monitor the runoff and output concentrations of nitrogen and phosphorus in each rainfall process. Two experimental indexes are set, respectively, the efficiency of searching particles in the solution space and the simplicity of calculating evolutionary operators in a single population. The main experimental schemes are as follows:

Step 1: Input 6 equations of the experiment into the experimental database;

Step 2: Use the four methods and the design method to calculate the equation;

Step 3: Calculate one by one. After each calculation iteration is completed, the next calculation will be carried out to improve the accuracy of experimental results.

Step 4: After the calculation, output the experimental results, and generate the experimental results through the above experimental platform. 


\section{Experimental Results}

Compare this method, Newton method and particle swarm optimization method to verify the convergence of the three methods, as shown in Fig. 7.

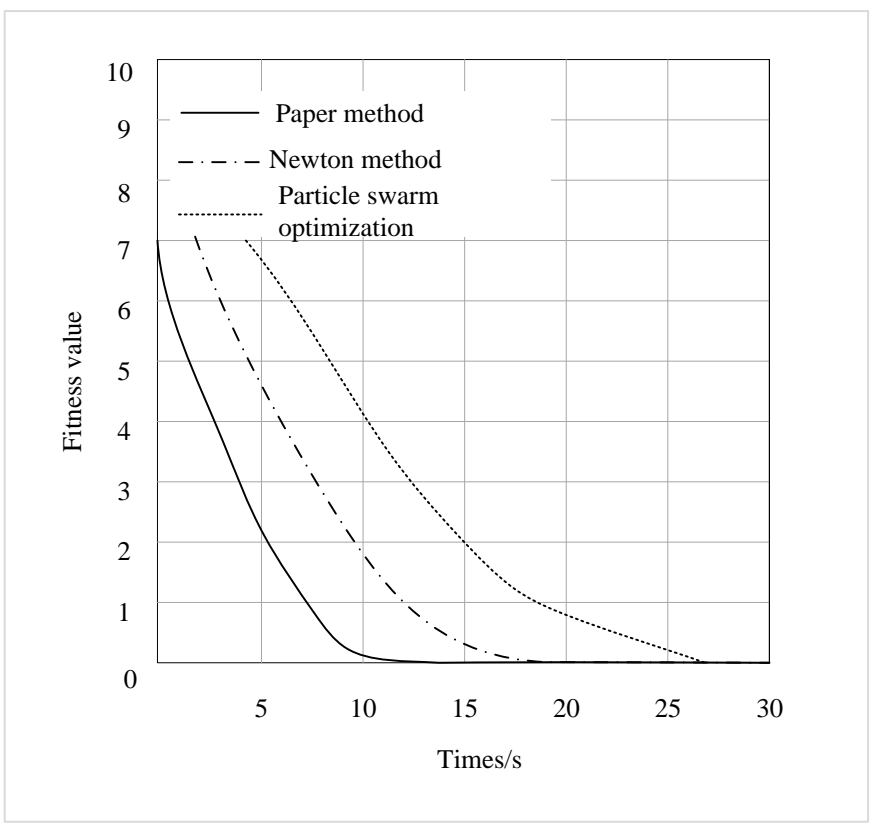

Fig. 7 comparison results of convergence

According to Fig. 7, the convergence speed of this paper is faster than that of other methods, which shows that the convergence effect of this method is good and can quickly converge to obtain the optimal solution of the nonlinear equation.

Will this paper method and Newton method, quasi-newton method and comparison of particle swarm optimization (PSO), multiple test, the test solution space in search of particle detection time, when measured in the same formula, different detection time response of different detection efficiency, through the experiment, four methods of experimental results, the method for design method of the comparison results are shown in Fig. 8:

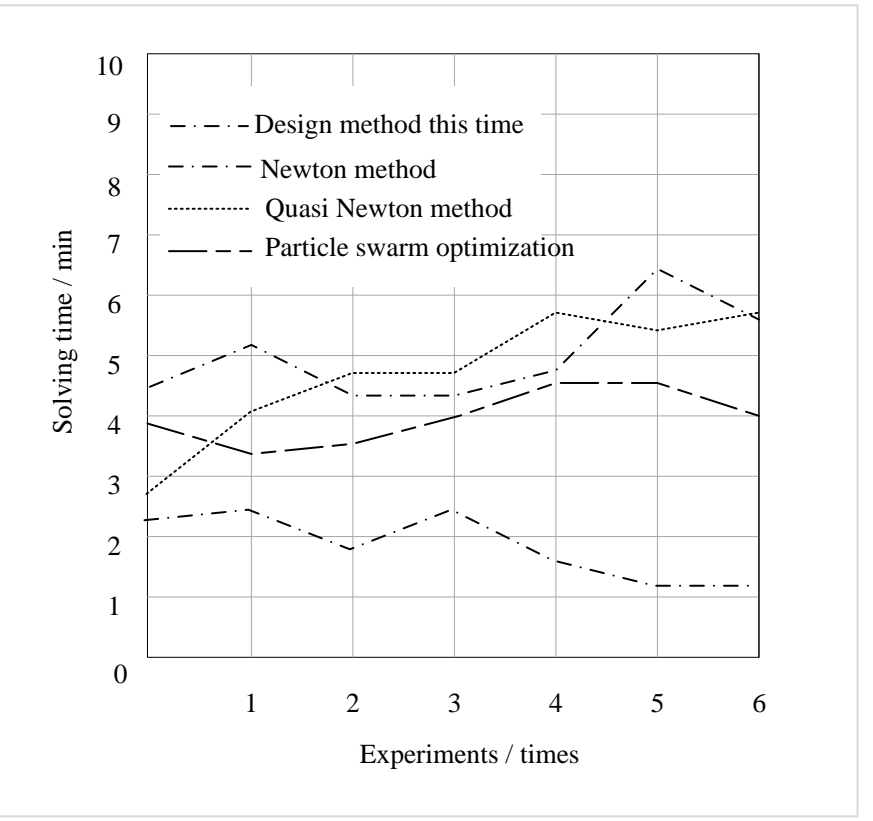

Fig. 8 Experimental comparison results

According to the comparison results in Fig. 8, in the process of 6 experiments, this design method has the shortest solution time than the traditional method. Therefore, through the above experiments, it can be proved that the optimal path analysis method for solving nonlinear equations with limited local error is more effective than the traditional method. And has the following advantages: good universality, can use a unified solution strategy to solve the nonlinear equations, programming workload is small; Can deal with complex constraint solving cases; Initial iteration values are not required.

The calculation simplicity of evolutionary operators in a single population is affected by the number of evolutionary operators and search distance. The method proposed in this paper is compared with Newton method, quasi-newton method and particle swarm optimization algorithm. The calculation simplicity test results of evolutionary operators are shown in Fig. 9. 


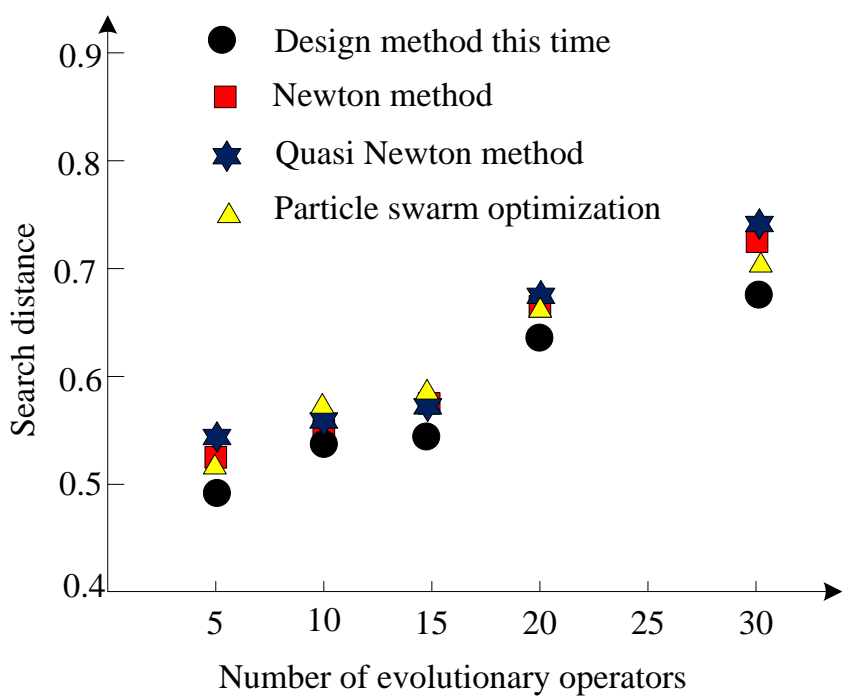

Fig. 9 Test results of computational simplicity of evolutionary operators

As shown in Fig. 9, under the different evolutionary operator number limitation, compared with other methods, the method of searching the shortest distance that is within the shortest distance can realize optimization particles of the evolution operator, to illustrate the calculation of the method is simple, which can realize the equations involved in the petroleum exploration and engineering example is simple operation, and is applied in practice.

\section{DISCUSSION}

Aiming at the problems of time-consuming and poor effect of traditional methods for solving nonlinear equations, an optimal path analysis method for solving nonlinear equations with limited local error is proposed and designed. According to the established constraints, the optimal path to solve the nonlinear equations under the condition of local error is given. The nonlinear equation optimization path model is obtained by solving the local error limit. The solution of nonlinear optimization problem is an important part of optimization problem research in real life. In practice, the accuracy of solving one-dimensional subproblems needs to be improved, because one-dimensional accurate line search method will produce a lot of computation, especially when the initial point is far from the optimal solution point. For the finite local error condition, the optimal objective setting of solving the nonlinear equation is set. If the number of subsets of in is infinite, a certain empirical risk of the local error condition is taken as the training object of displacement calculation until the optimal solution is obtained. Because the quantum probability amplitude indicates that the quantum chromosome carries information about multiple states, the quantum chromosome is in the superposition state of multiple determined states before we measure it. The solution is either to design special evolutionary operators or to modify invalid chromosomes after evolution, both of which increase the additional cost of the program. In this case, the evolutionary operation of quantum chromosome is a good strategy. Therefore, through the above analysis, the design and application conditions of genetic quantum algorithm are determined.

Under the condition of the effectiveness of the optimization path analysis method for solving finite nonlinear equations, the local error process of the design is theoretically verified. The proposed method is compared with Newton method, quasi Newton method and particle swarm optimization (PSO), multiple tests, and the particle detection time is found in the trial solution space. When measured in the same formula, Different detection time response different detection efficiency, through the experiment, the experimental results of the four methods, the comparison results of the design method. Under different restrictions on the number of evolutionary operators, compared with other methods, the method of searching the shortest distance within the shortest distance can realize the optimized particles of evolutionary operators, which shows that the method is simple in calculation, can realize the equations and simple operations involved in petroleum exploration and engineering examples, and has been applied in practice.

\section{CONCLUSION}

Optimization method is an important research branch of operations research and control theory. In practical problems, nonlinear optimization problems are often encountered. The hybrid quasi-Newton algorithm and particle swarm optimization algorithm are beneficial to enrich the search behavior of the optimization process, enhance the ability and efficiency of the hybrid algorithm, and obtain high-quality optimal solutions. The hybrid algorithm of quasi-Newton method and particle swarm optimization algorithm overcomes the shortcomings of each algorithm and eliminates the limitation of the previous single use. Compared with other single optimization algorithms, the search efficiency of the particle swarm quasi-Newton hybrid algorithm is very high. The particle swarm optimization (PSO) algorithm provides a good initial point for the global convergence of the quasi-Newton method, so that the quasi-Newton method can give Giving full play to its strong local search capabilities, it can be organically integrated with particle swarm optimization (PSO) algorithms to the greatest extent.

But there are some problems with this research. In future research, improvements are still needed in many aspects. Since the development of particle swarm algorithm is not perfect, it is the choice of initial point. The choice of initial point of particle swarm has a certain impact on the performance of the algorithm, although particle swarm algorithm is the initial point. There is no special requirement, but in order to make the initial population cover the entire search space as evenly as possible and improve the global search capability, certain restrictions on the choice of initial points are required. Another aspect is the choice of parameters. The parameters of the particle swarm optimization algorithm mainly include the maximum speed, two 
acceleration constants, inertia constant or shrinkage factor, etc. The reasonable choice of each parameter has a great influence on the solution result.

\section{References}

[1] H. H. Zhang, J. Z. Su, Q. Y. Wang, Y. M. Liu, L. Good, and J. M. Pascual, "Predicting seizure by modeling synaptic plasticity based on EEG signals - A case study of inherited epilepsy," Communications in Nonlinear Science and Numerical Simulation, vol. 56, no. 12, pp. 330-343, 2017.

[2] M. G. Abdol, and V. Azam, "Applications of artificial neural networks for adsorption removal of dyes from aqueous solution: A review," Advances in Colloid and Interface Science, vol. 245, no. 6, pp. 20-39, 2017.

[3] R. Rostamian, and H. Behnejad, "Insights into doxycycline adsorption onto graphene nanosheet: A combined quantum mechanics, thermodynamics, and kinetic study," Environmental Science and Pollution Research, vol. 25, no. 4, pp. 1-10, 2017.

[4] A. Patra, J. E. Bates, J. W. Sun, and J. P. Perdew, "Properties of real metallic surfaces: Effects of density functional semilocality and van der Waals nonlocality," Proceedings of the National Academy of Sciences, vol. 114, no. 44, pp. 9188-9192, 2017.

[5] W. Hamouche, C. Maurini, S. Vidoli, and A. Vincenti, "Multiparameter actuation of a neutrally-stable shell: A flexible gear-less motor," Proceedings of The Royal Society A Mathematical Physical and Engineering Sciences, vol. 473, no. 2204, pp. 20170364, 2017.

[6] O. Zavgorodnya, C. A. Carmona-Moran, V. Kozlovskaya, F. Liu, T. M. Wick, and E. Kharlampieva, "Temperature-responsive nanogel multilayers of poly (N-vinylcaprolactam) for topical drug delivery," Journal of Colloid and Interface Science, vol. 506, no. 5, pp. 589-602, 2017.

[7] S. Gerhard, and H. Peter, "A non-equilibrium approach to allosteric communication," Philosophical Transactions of The Royal Society B Biological Sciences, vol. 373, no. 1749, pp. 20170187, 2018.

[8] L. Pu, R. Saraf, and V. Maheshwari, "Bio-inspired interlocking random 3-D structures for tactile and thermal sensing," Scientific Reports, vol. 7, no. 1, pp. 5834, 2017.

[9] C. S. Zhou, C. Zhang, D. Tian, K. Wang, M. Z. Huang, and Y. B. Liu, "A software sensor model based on hybrid fuzzy neural network for rapid estimation water quality in Guangzhou section of Pearl River, China," Journal of Environmental Science and Health, Part A. Toxic/hazardous Substances \& Environmental Engineering, vol. 53, no. 1, pp. 1-8, 2107.

[10] C. Reddick, M. Sorin, H. Sapoundjiev, and Z. Aidoun, "Effect of a mixture of carbon dioxide and steam on ejector performance: An experimental parametric investigation," Experimental Thermal \& Fluid Science, vol. 92, no. 3, pp. 353-365, 2017.

[11] C. S. Nelson, T. Huffman, J. A. Jenks, E. C. D. L. Rosa, G. H. Xie, N. A. Vandergrift, R. F. Pass, J. Pollara, and S. Permar, "HCMV glycoprotein B subunit vaccine efficacy mediated by nonneutralizing antibody effector functions," Proceedings of the National Academy of Sciences, vol. 115, no. 24, pp. 201800177, 2018.

[12] K. S. Bhat, R. Ahmad, J. Y. Yoo, and Y. B. Hahn, "Fully nozzle-jet printed non-enzymatic electrode for biosensing application," Journal of Colloid and Interface Science, vol. 512, no. 23, pp. 480-488, 2017.

[13] V. Pansare, V. Hwang, C. Figueroa, and R. K. Prud'homme, "Adsorption characteristics of charged and nonionic small molecules to colloidal alumina," Journal of Colloid and Interface Science, vol. 512, no. 5, pp. 29-38, 2017.

[14] D. Lis, T. Stellingwerff, C. M. Kitic, J. Fell, and K. D. K. Ahuja, "Low FODMAP: A preliminary strategy to reduce gastrointestinal distress in athletes," Medicine \& Science in Sports \& Exercise, vol. 50, no. 1, pp. 1, 2017.

[15] B. Suanet, and T. C. Antonucci, "Cohort differences in received social support in later life: the role of network type," The Journals of Gerontology. Series B, Psychological Sciences and Social Sciences, vol. 72, no. 4, pp. 75, 2017.

[16] B. C. Simionescu, M. Drobota, D. Timpu, T. Vasiliu, C. A. Constantinescu, D. Rebleanu, M. Calin, and G. David, "Biopolymers/poly (ع-caprolactone)/polyethylenimine functionalized nano-hydroxyapatite hybrid cryogel: Synthesis, characterization and application in gene delivery," Materials Science and Engineering, vol. 81, no. 12, pp. 167-176, 2017.

[17] M. Toshiaki, "Camera calibration based on the principal rays model of imaging optical systems," Journal of the Optical Society of America A - Optics Image Science and Vision, vol. 34, no. 4, pp. 624, 2017.

[18] T. Minamitani, Y. J. Ma, H. F. Zhou, H. Kida, C. Y. Tsai, M. Obana, D. Okuzaki, Y. Fujio, A. Kumanogoh, B. Zhao, H. Kikutani, E. Kieff, B. E. Gewurz, and T. Yasui, "Mouse model of Epstein-Barr virus LMP1- and LMP2A-driven germinal center B-cell lymphoproliferative disease," Proceedings of the National Academy of Sciences of the United States of America, vol. 114, no. 7, pp. 4751, 2017.

[19] R. S. Prakash, A. Sinha, G. Tomar, and R. V. Ravikrishna, "Liquid jet in crossflow - Effect of liquid entry conditions," Experimental Thermal \& Fluid Science, vol. 93, pp. 45-56, 2017.

[20] S. Yuan, J. S. Qin, and H. Q. Xu, “[Ti 8 Zr 2 O 12 (COO) 16] Cluster: An ideal inorganic building unit for photoactive metal-organic frameworks," ACS Central Science, vol. 4, no. 1, pp. 105-111, 2017.

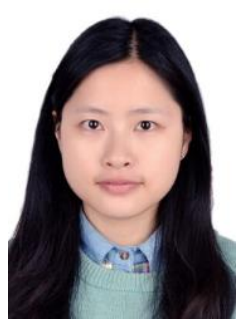

Xiaoxiao Ma, female, was born in May 1990. Her professional 
title is lecturer. She received her bachelor's degree in agricultural resources and environment from Huazhong Agricultural University in 2012. She received her master's degree in plant nutrition from Zhejiang University in 2015. She now works at Chongqing Vocational College of Transportation. Her areas of research include applied mathematics. She has published five academic papers. She is also involved in three research projects.

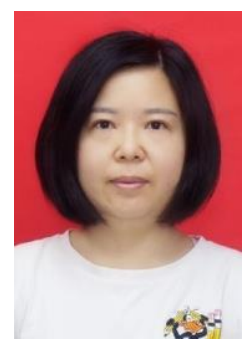

Xiaojuan Chen, female, was born in December 1986. Her professional title is lecturer. She received a bachelor's degree in horticulture from Jilin University in 2010. She received a PH.D. in horticulture and vegetable science from Zhejiang University in 2015. She is now working in Chongqing Vocational College of Transportation. Her field of study includes ideological and political education. She has published four academic papers. She is also involved in three research projects.

\section{Author Contributions:}

Xiaoxiao Ma established the optimization objective function of nonlinear equations. Xiaojuan Chen solved the optimal solution of nonlinear equations.

\section{Creative Commons Attribution License 4.0 (Attribution 4.0 International, CC BY 4.0)}

This article is published under the terms of the Creative Commons Attribution License 4.0 https://creativecommons.org/licenses/by/4.0/deed.en_US 